\title{
COMPLEX CONVEXITY AND FINITELY ADDITIVE VECTOR MEASURES
}

\author{
GORAZD LEŠNJAK
}

(Communicated by William J. Davis)

\begin{abstract}
We prove that complex uniform convexity of a complex normed space $X$ is equivalent to complex uniform convexity of bva $(\mathcal{F}, X)$. Similar results hold also for complex uniform convexity at a point. Some consequences for duals of certain operator spaces are discussed.
\end{abstract}

Introduction. One of the achievements in geometry of duals of operator spaces is the result of Ruess and Stegall characterizing extreme points of unit balls of $K(X, Y)^{*}[\mathbf{1 0}]$. Here $X$ and $Y$ are real Banach spaces and $K(X, Y)$ is the space of all compact linear operators from $X$ into $Y$. They have shown that ext $B\left(K(X, Y)^{*}\right)=$ ext $B\left(X^{* *}\right) \otimes \operatorname{ext} B\left(Y^{*}\right)$, where we write ext $B(Z)$ for the set of all extreme points of the unit ball $B(Z)$ of $Z$. For $x^{* *} \in X^{* *}$ and $y^{*} \in Y^{*}$ the functional $x^{* *} \otimes y^{*}$ in $K(X, Y)^{*}$ is defined by $\left(x^{* *} \otimes y^{*}\right)(T)=x^{* *}\left(T^{*} y^{*}\right)$ for every $T$ from $K(X, Y)$. The same result is valid for complex Banach spaces (see [7]). Other classes of special points in dual unit balls were also studied in [9].

For complex normed spaces Thorp and Whitley [12] introduced the notion of complex extreme point: an element $x$ in the unit sphere $S(X)$ of a complex normed space $X$ is called complex extreme if from $\|x+\lambda y\| \leq 1$ for every $\lambda \in \bar{\Delta}=$ $\{z \in \mathbf{C} ;|z| \leq 1\}$ it follows that $y=0$. The question of characterizing complex extreme points of $B\left(K(X, Y)^{*}\right)$ seems to be still open in general. In the case $X=Y=\not$, where $\not$ is a Hilbert space, the answer was given by Mattila [8] and, in the more general setting of $C^{*}$-algebras, by Haagerup (see [1]). The dual of $K(\not H)$ is complex uniformly convex and thus every point of $S\left(K(\not{H})^{*}\right)$ is complex extreme. The normed space $X$ is said to be complex uniformly convex if for any $\varepsilon>0$ there exists a $\delta>0$ such that from $y \in X,\|y\| \geq \varepsilon$ and $\|x+\lambda y\| \leq 1$ for every $\lambda \in \bar{\Delta}$ it follows that $\|x\| \leq 1-\delta$. The notion of complex uniform convexity was introduced by Globevnik [5]. Various kinds of moduli of complex uniform convexity were defined in [1]. For complex normed spaces all these moduli are equivalent in a certain sense as shown in [3]. As a consequence, uniform PL-convexity, uniform $H_{\infty}$-convexity (introduced in [1]) and complex uniform convexity are equivalent properties of a space. From [6] or by combination of results in [1 and 3] we know that complex uniform convexity of $L_{1}(\mu, X)$ is equivalent to that of $X$ for any nonnegative measure $\mu$. The aim of this note is to present some results on $K(X, Y)^{*}$,

Received by the editors December 24, 1986.

1980 Mathematics Subject Classification (1985 Revision). Primary 46B20; Secondary 46G10, 46M05, 47D15.

Key words and phrases. Vector measures, compact operators, complex extreme points, tensor products.

This work was supported by the Research Council of Slovenia. 
where $X$ is an AL-space and $Y$ is either an AL-space or a space with complex uniformly convex dual. Definitions and properties of AL-spaces and AM-spaces can be found in Schaefer [11].

1. Complex convexity and finitely additive measures. Let us denote by $\Omega$ a fixed nonempty set, by $\mp$ an algebra of subsets of $\Omega$ and by $X$ a normed space over $\mathbf{C}$. We shall write bva $(\mathcal{F}, X)$ for the space of all finitely additive set functions defined on $\mathcal{F}$ and with values in $X$ which have finite variation: $\varphi \in \operatorname{bva}(\mathcal{F}, X)$ if and only if

$$
|\varphi|(\Omega)=\sup \left\{\sum_{k \in I}\left\|\varphi\left(E_{k}\right)\right\| ;\left\{E_{k}\right\}_{k \in I}\right. \text { is a finite family }
$$

$$
\text { of disjoint elements of } ₹\}<\infty \text {. }
$$

$|\varphi|(\Omega)$ defines a norm on the vector space bva $(\mathcal{F}, X)$ and this space is complete if and only if $X$ is a Banach space $[2,4]$. To compute the norm of $\varphi \in \operatorname{bva}(\mathcal{F}, X)$ it is enough to take the supremum over all finite partitions of $\Omega$ with elements in $\mathcal{F}$. In the case $X=\mathbf{C}$ we simply write bva $(\mathcal{F})$.

PROPOSITION 1.1. The normed space $X$ is complex uniformly convex if and only if the space bva $(\mathcal{F}, X)$ is complex uniformly convex.

PROOF. The complex uniform convexity of $X$ is equivalent to that of $l_{1}(X)$ (see [6]). Suppose that $X$ is complex uniformly convex. It follows that for every $\varepsilon>0$ the modulus of complex uniform convexity $c(\varepsilon)$ of $l_{1}(X)$, defined as

$$
\begin{aligned}
& c(\varepsilon)=\inf \{1-\|f\| ;\|f+\lambda g\| \leq 1 \text { for every } \lambda \in\{1, i,-1,-i\}, \\
& \left.\qquad f \in l_{1}(X), g \in l_{1}(X),\|g\| \geq \varepsilon\right\},
\end{aligned}
$$

is positive. Take $\varphi$ and $\psi \neq 0$ from bva $(\mathcal{F}, X)$ such that for every $\lambda \in\{1, i,-1,-i\}$ the inequality $|\varphi+\lambda \psi|(\Omega) \leq 1$ is valid. Denote $|\psi|(\Omega)=\varepsilon>0$. Then for any $n \in \mathbf{N}$ we have a partition $P_{n}$ of $\Omega$ such that

$$
\sum_{P_{n}}\left\|\psi\left(E_{k}\right)\right\|>\varepsilon\left(1-2^{-n}\right) .
$$

Fix a partition $P$ and write $R_{n}$ for the common refinement of $P$ and $P_{n}$. For every $\lambda \in\{1, i,-1,-i\}$ the inequalities

$$
\sum_{R_{n}}\left\|\varphi\left(F_{k}\right)+\lambda \psi\left(F_{k}\right)\right\| \leq 1 \text { and } \sum_{R_{n}}\left\|\psi\left(F_{k}\right)\right\|>\varepsilon\left(1-2^{-n}\right)
$$

are valid. If we consider the sequences $\left\{\varphi\left(F_{k}\right)\right\}$ and $\left\{\psi\left(F_{k}\right)\right\}$ as elements of $l_{1}(X)$ the complex uniform convexity of this space implies

$$
\sum_{R_{n}}\left\|\varphi\left(F_{k}\right)\right\| \leq 1-c\left(\varepsilon\left(1-2^{-n}\right)\right) .
$$

Hence $\sum_{P}\left\|\varphi\left(G_{k}\right)\right\| \leq 1-c\left(\varepsilon\left(1-2^{-n}\right)\right)$ for every $n \in \mathbf{N}$, which yields that

$$
|\varphi|(\Omega) \leq 1-\sup \left\{c\left(\varepsilon\left(1-2^{-k}\right)\right) ; k \in \mathbf{N}\right\} .
$$

The converse implication is straightforward. 
COROLlaRY 1.2. (i) The space bva $(\mathcal{F})$ is complex uniformly convex.

(ii) For a compact Hausdorff space $K$ the space $M(K)$ of all countably additive Borel measures on $K$ is complex uniformly convex. Also, bva $(\mathcal{F}, M(K))$ is complex uniformly convex.

(iii) For any measure space $(\Omega, \Sigma, \mu)$ and every $p$, where $1 \leq p<\infty$, the space $\operatorname{bva}\left(\mathcal{F}, L_{p}(\mu)\right)$ is complex uniformly convex.

In the next proposition we shall confine ourselves to the case $X=C(K)$, the space of continuous functions on a compact Hausdorff space $K$ equipped with sup norm. For each $A \in \mathcal{F}$ and $\varphi \in \operatorname{bva}(\mathcal{F}, C(K))$ the value $\varphi(A)$ is a continuous function on $K$. For every $t \in K$ we define $\varphi_{t}: \mathcal{F} \rightarrow \mathbf{C}$ by $\varphi_{t}(A)=\varphi(A)(t)$ for $A \in \mathcal{F}$. Then $\varphi_{t}$ is an element of bva $(\mathcal{F})$ and $\left|\varphi_{t}\right|(\Omega) \leq|\varphi|(\Omega)$.

Proposition 1.3. A measure $\varphi \in S(\mathrm{bva}(\mathcal{F}, C(K)))$ is a complex extreme point if and only if there exists a dense subset $L$ of $K$ such that for every $t \in L$ we have $\left|\varphi_{t}\right|(\Omega)=1$.

ProOF. Suppose that $\varphi$ and $\psi$ are elements of $\operatorname{bva}(\mathcal{F}, C(K))$ such that for every $\lambda \in \bar{\Delta}$ we have $|\varphi+\lambda \psi|(\Omega) \leq 1$. This implies that for every $t \in L$ from $\left|\varphi_{t}\right|(\Omega)=1$ it follows that $\left|\varphi_{t}+\lambda \psi_{t}\right|(\Omega)=1$ for every $\lambda \in \bar{\Delta}$. Thus, by Corollary 1.2 , we have $\psi_{t} \equiv 0$ and, therefore, for every $A \in \mathcal{F}$ the continuous function $\psi(A)$ is zero at all points of $L$. Hence $\psi(A)=0$ and consequently $\psi \equiv 0$.

For the converse suppose that there exists a point $t_{0} \in K$ and a neighbourhood $U$ of $t_{0}$ such that for every $t \in U$ we have $\left|\varphi_{t}\right|(\Omega)<1$. Then there is a continuous function $f$ on $K$ with the properties: $0 \leq f(t) \leq 1, f\left(t_{0}\right)=1$ and $\left.f\right|_{(K-U)}=0$. The construction of $\psi$ now goes as follows: for every $A \in \mathcal{F}$ and $t \in K$ we define

$$
\psi(A)(t)=\left(|\varphi|(A)-\left|\varphi_{t}\right|(A)\right) \cdot f(t) .
$$

For any $A$ in $\mathcal{F}$ and $\lambda \in \bar{\Delta}$ we have

$$
\begin{aligned}
\|(\varphi+\lambda \psi)(A)\| & =\max \{|(\varphi+\lambda \psi)(A)(t)| ; t \in K\} \\
& =\max \left\{\max _{t \in U^{c}}|\varphi(A)(t)|, \sup _{s \in U}|\varphi(A)(s)+\lambda \psi(A)(s)|\right\} .
\end{aligned}
$$

On one hand it holds that

$$
\max _{t \in U^{c}}|\varphi(A)(t)| \leq \max _{t \in K}|\varphi(A)(t)|=\|\varphi(A)\| \leq|\varphi|(A),
$$

while on the other we see that

$$
\begin{aligned}
|\varphi(A)(s)+\lambda \psi(A)(s)| & =\left|\varphi(A)(s)+\lambda f(s)\left(|\varphi|(A)-\left|\varphi_{s}\right|(A)\right)\right| \\
& \leq|\varphi(A)(s)|+|\varphi|(A)-\left|\varphi_{s}\right|(A) \\
& =|\varphi|(A)-\left(\left|\varphi_{s}\right|(A)-|\varphi(A)(s)|\right) \leq|\varphi|(A) .
\end{aligned}
$$

Therefore, $\|(\varphi+\lambda \psi)(A)\| \leq|\varphi|(A)$ and because of finite additivity of $|\varphi|$ we obtain for every finite partition $P$ of $\Omega$ the inequality

$$
\sum_{P}\left\|(\varphi+\lambda \psi)\left(E_{k}\right)\right\| \leq \sum_{P}|\varphi|\left(E_{k}\right)=|\varphi|(\Omega)=1,
$$

which yields $|\varphi+\lambda \psi|(\Omega) \leq 1$ and $\psi$ is not a complex extreme point. 
REMARK. Let $F$ be a complex AM-space with unit and $K$ the $\sigma\left(F^{*}, F\right)$-compact set of all lattice homomorphisms of norm 1 on $F$. It is known [11] that $F$ is isometrically isomorphic to $C(K)$. In this case the above proposition characterizes complex extreme points of $S($ bva $(\mathcal{F}, F))$.

Let $X$ be a complex normed space and $x \in S(X)$. Then we define the modulus of complex uniform convexity of $X$ at $x$ for every $\varepsilon \geq 0$ by

$$
\delta_{x}(\varepsilon)=\inf \left\{\sum_{k=0}^{3}\left\|x+i^{k} y\right\|-4 ; y \in X,\|y\| \geq \varepsilon\right\}
$$

(see also [1]). Similarly, as in [6] it can be shown that the function $t \rightarrow \delta_{x}(t) / t$ is nondecreasing for positive $t$. The following definition is limited to the purposes of this paper.

DEFInItion. A normed space $X$ is complex uniformly convex at $x \in S(X)$ if the modulus $\delta_{x}(\varepsilon)$ of complex uniform convexity of $X$ at $x$ is strictly positive for every $\varepsilon>0$.

We have already mentioned the equivalence of uniform PL-convexity and complex uniform convexity. Hence this property of a normed space $X$ implies that this space is complex uniformly convex at every point of $S(X)$. If $X$ is not finite dimensional, the converse does not hold in general. It is easy to see that a point $x \in S(X)$ is complex extreme if $X$ is complex uniformly convex at $x$. In spaces $C(K), K$ compact, the converse is also valid: these are complex uniformly convex at every complex extreme point.

Proposition 1.4. Let $X$ and $Y$ be complex normed spaces and $\|\cdot\|$ a crossnorm on $X \otimes Y$. If for $x \in S(X)$ and $y \in S(Y)$ the space $(X \otimes Y,\|\cdot\|)$ is complex uniformly convex at $x \otimes y$, then $X$ is complex uniformly convex at $x$ and $Y$ is complex uniformly convex at $y$.

ProOF. By the definition of cross-norm it follows that for every $s \in X, s \neq 0$,

$$
\sum_{k=0}^{3}\left\|x+i^{k} s\right\|-4=\sum_{k=0}^{3}\left\|x \otimes y+i^{k}(s \otimes y)\right\|-4 \geq \delta_{x \otimes y}(\|s\|) .
$$

Hence $\delta_{x}(\|s\|) \geq \delta_{x \otimes y}(\|s\|)$, which implies that $\delta_{x \otimes y}(\varepsilon) \leq \min \left\{\delta_{x}(\varepsilon), \delta_{y}(\varepsilon)\right\}$ for every $\varepsilon>0$.

In the case of finitely additive measures even more can be said.

THEOREM 1.5. A complex normed space $X$ is complex uniformly convex at $x \in S(X)$ if and only if for any $\alpha \in S(\mathrm{bva}(\mathcal{F}))$ the normed space bva $(\mathcal{F}, X)$ is complex uniformly convex at $x \otimes \alpha$.

ProOF. Let $X$ be complex uniformly convex at $x \in S(X)$ and take any $\psi \in$ $\operatorname{bva}(\mathcal{F}, X), \psi \neq 0$. Denote $\|\psi\|$ by $\varepsilon$. Then there exists a constant $C$ such that $0<C \leq 1$ and that for every $t \geq \varepsilon / 3$ we have $\delta_{x}(t) \geq C t$. Take an $\alpha$ from $S(\operatorname{bva}(\mathcal{F}))$ and choose a partition $P$ of $\Omega$ such that the estimates $\sum_{P}\left\|\psi\left(E_{k}\right)\right\|>2 \varepsilon / 3$ and 
$\sum_{P}\left|\alpha\left(E_{k}\right)\right|>1-C \varepsilon / 24$ are valid. Hence

$$
\begin{aligned}
\sum_{j=0}^{3} \mid x & \otimes \alpha+i^{j} \psi \mid(\Omega)-4+\frac{C \varepsilon}{6} \\
> & \sum_{j=0}^{3}\left(\sum_{\mathcal{P}}\left\|x \alpha\left(E_{k}\right)+i^{j} \psi\left(E_{k}\right)\right\|\right)-4 \sum_{\mathcal{P}}\left|\alpha\left(E_{k}\right)\right| \\
= & \sum_{\mathcal{P}}\left(\sum_{j=0}^{3}\left\|x \alpha\left(E_{k}\right)+i^{j} \psi\left(E_{k}\right)\right\|-4\left|\alpha\left(E_{k}\right)\right|\right) .
\end{aligned}
$$

Let us denote by $f\left(E_{k}\right)$ the value of

$$
\sum_{j=0}^{3}\left\|x \alpha\left(E_{k}\right)+i^{j} \psi\left(E_{k}\right)\right\|-4\left|\alpha\left(E_{k}\right)\right| .
$$

Let $A$ be the family of those $E_{k} \in P$ for which $\alpha\left(E_{k}\right)=0$, and let $B$ be the set of $E_{k} \in P$ such that $\alpha\left(E_{k}\right) \neq 0$ and $\left\|\psi\left(E_{k}\right)\right\| /|\alpha|\left(E_{k}\right) \geq \varepsilon / 3$. Define $C=$ $P-(A \cup B)$. It follows that $\sum_{c}\left\|\psi\left(E_{k}\right)\right\|<\varepsilon / 3 . \sum_{c}|\alpha|\left(E_{k}\right) \leq \varepsilon / 3$, and therefore $\sum_{A \cup B}\left\|\psi\left(E_{k}\right)\right\|>\varepsilon / 3$. For $E_{k}$ in $B$ we get

$$
\begin{aligned}
f\left(E_{k}\right) & =\left|\alpha\left(E_{k}\right)\right| \cdot\left(\sum_{j=0}^{3}\left\|x+\frac{i^{j} \psi\left(E_{k}\right)}{\alpha\left(E_{k}\right)}\right\|-4\right) \\
& \geq\left|\alpha\left(E_{k}\right)\right| \cdot \delta_{x}\left(\frac{\left\|\psi\left(E_{k}\right)\right\|}{\left|\alpha\left(E_{k}\right)\right|}\right) \geq C\left\|\psi\left(E_{k}\right)\right\| .
\end{aligned}
$$

Since for $E_{k}$ in $A$ it holds that $f\left(E_{k}\right)=4\left\|\psi\left(E_{k}\right)\right\|$, we obtain

$$
\begin{aligned}
\frac{C \varepsilon}{6}+ & \sum_{j=0}^{3}\left|x \otimes \alpha+i^{j} \psi\right|(\Omega)-4 \\
& >\sum_{P} f\left(E_{k}\right) \geq \sum_{A} f\left(E_{k}\right)+\sum_{B} f\left(E_{n}\right) \\
& \geq C \sum_{A \cup B}\left\|\psi\left(E_{k}\right)\right\|>\frac{C \varepsilon}{3},
\end{aligned}
$$

or equivalently

$$
\sum_{j=0}^{3}\left|x \otimes \alpha+i^{j} \psi\right|(\Omega)-4>\frac{C \varepsilon}{6} .
$$

REMARK. For any normed spaces $X$ and $Y$ the following holds: If $x \otimes y$ is the complex extreme point of $S(X \otimes Y)$, where $X \otimes Y$ is normed with a cross-norm, then $x$ and $y$ are complex extreme. Theorem 1.5 shows that partial converse holds if $x$ is complex extreme in a special way and $Y=\operatorname{bva}(\mathcal{F})$.

2. Complex convexity in duals of certain operator spaces. In this section we shall use the following result about representation of continuous vector-valued functions. Let $\Omega$ be a compact Hausdorff space, $S$ the $\sigma$-algebra of Borel subsets of 
$\Omega$ and $Z$ a Banach space. By $\operatorname{rcabv}(S, Z)$ we denote the subspace consisting of all regular countably additive vector measures in bva $(S, Z)$. Singer proved (see [2, p. 182]) that the dual of $C(\Omega, Z)$ is isometrically isomorphic to $\operatorname{rcabv}\left(S, Z^{*}\right)$. Denote the measure corresponding to $\varphi \in C(\Omega, Z)^{*}$ by $\mu_{\varphi}$. The isomorphism is then given by $\varphi(f)=\int_{\Omega} f d \mu_{\varphi}$ for every $f$ in $C(\Omega, Z)$.

This representation will help us to get some results about complex convexity in duals of certain operator spaces.

Let $E$ be an AL-space with dual $E^{*}$. This is an AM-space with unit [11]. Denote by $K$ the $\sigma\left(E^{* *}, E^{*}\right)$-compact set of all lattice homomorphisms of norm 1 on $E$ and by $\mp$ the $\sigma$-algebra of Borel subsets of $K$.

Proposition 2.1. Let $E, K$, and $\mathcal{F}$ be as above and $X$ be any Banach space. Then $K(E, X)^{*}$ is isometrically isomorphic to $\operatorname{rcabv}\left(\mathcal{F}, X^{*}\right)$.

ProOF. AM-space $E^{*}$ has metric approximation property [11], hence $K(E, X)$ is isometrically isomorphic to $E^{*} \otimes_{\varepsilon} X[\mathbf{2}]$. Taking into account that $E^{*}$ is isometrically isomorphic to $C(K)$, it follows that the next chain of isometric isomorphisms is valid:

$$
K(E, X) \cong E^{*} \otimes_{\varepsilon} X \cong C(K) \otimes_{\varepsilon} X \cong C(K, X) .
$$

Using this and the above representation we get the result.

For every $\phi \in K(E, X)^{*}$ there exists a regular countably additive vector measure $\varphi$, defined on Borel subsets of $K$ and with values in $X^{*}$ such that $\|\phi\|=\|\varphi\|$. If we identify $A \in K(E, X)$ with the appropriate function $f_{A}$ in $C(K, X)$ in the sense of the above mentioned isomorphisms, then the value of $\phi$ at $A$ is given by $\phi(A)=\int_{K} f_{A} d \varphi$.

Let us take $E=l_{1}$ as an illustration. Then $E^{*} \cong l_{\infty} \cong C(K)$ for the appropriate $K$. Consequently $K\left(l_{1}, X\right)^{*} \cong \operatorname{rcabv}\left(\mathcal{F}, X^{*}\right)$. On the other hand, it can be proved directly that $K\left(l_{1}, X\right)^{*} \cong \operatorname{bva}\left(P \mathrm{~N}, X^{*}\right)$. For every $\phi \in K\left(l_{1}, X\right)^{*}$ there exists an element $\mu_{\phi} \in \operatorname{bva}\left(P \mathbf{N}, X^{*}\right)$ such that $\|\phi\|=\left|\mu_{\phi}\right|(\mathbf{N})$ and $\phi(A)=\int_{\mathbf{N}} a d \mu_{\phi}$ where an element $A \in K\left(l_{1}, X\right)$ is identified with the corresponding element $a$ in $l_{\infty} \otimes_{\varepsilon} X$.

COROLlary 2.2. (i) Let $E$ be a complex AL-space and $X$ a complex Banach space with complex uniformly convex dual $X$. Then $K(E, X)^{*}$ is complex uniformly convex.

(ii) Let $E$ and $F$ be complex $A L$-spaces. By $L$ we denote the $\sigma\left(F^{* *}, F^{*}\right)$-compact set of all lattice homomorphisms of norm 1 on $F$. Then we identify $K(E, F)^{*}$ with $\operatorname{rcabv}(\mathcal{F}, C(L))$. Hence it follows that a point in $S\left(K(E, F)^{*}\right)$ is complex extreme iff for the corresponding measure $\varphi$ in $S(\operatorname{rcabv}(\mathcal{F}, C(L)))$ there exists a dense subset $L_{\varphi}$ of $L$ such that, for every $t \in L_{\varphi},\left|\varphi_{t}\right|(K)=1$.

Proposition 2.3. Let $X$ and $Y$ be Banach spaces. If $Y$ is reflexive, then $L(X, Y)$ is isometrically isomorphic to $L\left(Y^{*}, X^{*}\right)$ and $K(X, Y)$ is isometrically isomorphic to $K\left(Y^{*}, X^{*}\right)$.

ProOF. It is known that $A \rightarrow A^{*}$ is injective and an isometric linear map from $L(X, Y)$ into $L\left(Y^{*}, X^{*}\right)$, or from $K(X, Y)$ into $K\left(Y^{*}, X^{*}\right)$. To prove surjectivity we pick an operator $B \in L\left(Y^{*}, X^{*}\right)$ and show that $B=A^{*}$ for $A$ defined by $A=j^{-1} \circ B^{*} \circ i$. Here we denote by $i$ and $j$ the canonical embeddings of $X$ into 
$X^{* *}$ and $Y$ into $Y^{* *}$, respectively. If $Y$ is reflexive then $j^{-1}$ is well defined, and, if $B \in K\left(Y^{*}, X^{*}\right)$, then $A \in K(X, Y)$. For every $y^{*}$ in $Y^{*}$ and $x$ in $X$ it follows that

$$
\begin{aligned}
\left(A^{*}\left(y^{*}\right)\right)(x) & =\left(\left(j^{-1} \circ B^{*} \circ i\right)^{*}\left(y^{*}\right)\right)(x) \\
& =y^{*}\left(\left(j^{-1} \circ B^{*} \circ i\right)(x)\right)=y^{*}\left(\left(j^{-1} \circ B^{*}\right)(i(x))\right) \\
& =j\left(\left(j^{-1} \circ B^{*}\right)(i(x))\right)\left(y^{*}\right) \\
& =\left(B^{*}(i(x))\right)\left(y^{*}\right)=(i(x))\left(B y^{*}\right)=\left(B y^{*}\right)(x) .
\end{aligned}
$$

COROLLARY 2.4. (i) Let $X$ be a Banach space and $Y$ a reflexive Banach space. Then $L(X, Y)^{*} \cong L\left(Y^{*}, X^{*}\right)^{*}$ and $K(X, Y)^{*} \cong K\left(Y^{*}, X^{*}\right)^{*}$.

(ii) Let $E$ be a complex $A L$-space and $Y$ a reflexive complex Banach space with complex uniformly convex dual.

Then $K\left(Y^{*}, E^{*}\right)^{*}$ is complex uniformly convex.

Let us take $E=l_{1}$ again. Then for $Y$ reflexive with complex uniformly convex dual the space $K\left(Y^{*}, l_{\infty}\right)^{*}$ is complex uniformly convex. In particular, for every measure space $(\Omega, \Sigma, \mu)$ and every $p \in(1, \infty)$ the space $K\left(L_{p}(\mu), l_{\infty}\right)^{*}$ is complex uniformly convex.

\section{BIBLIOGRAPHY}

1. W. J. Davis, D. J. H. Garling and N. Tomczak-Jaegermann, The complex convexity of quasinormed linear spaces, J. Funct. Anal. 55 (1984), 110-150.

2. J. Diestel and J. J. Uhl, Vector measures, Math. Surveys, no. 15, Amer. Math. Soc., Providence, R. I., 1977.

3. S. J. Dilworth, Complex convexity and the geometry of Banach spaces, Math. Proc. Cambridge Philos. Soc. 99 (1986), 495-506.

4. N. Dunford and J. Schwartz, Linear operators, Part I, Interscience, New York, 1958.

5. J. Globevnik, On complex strict and uniform convexity, Proc. Amer. Math. Soc. 47 (1975), 175-178.

6. V. M. Kadec, On complex uniform convexity of the Lebesgue-Bochner spaces, Teor. Funktsiǔ Funktsional Anal. Prilozhen 40 (1983), 71-74. (Russian)

7. A. Lima and G. Olsen, Extreme points in duals of complex operator spaces, Proc. Amer. Math. Soc. 94 (1985), 437-440.

8. K. Mattila, Complex strict and uniform convexity and hyponormal operators, Math. Proc. Cambridge Philos. Soc. 96 (1984), 483-493.

9. W. Ruess, Duality and geometry of spaces of compact operators, Proc. 3rd Paderborn Conf. Funct. Anal. (1983), North-Holland Math. Studies, vol. 90, 1984, pp. 59-78.

10. W. Ruess and C. Stegall, Extreme points in duals of operator spaces, Math. Ann. 261 (1982), 535-546.

11. H. H. Schaefer, Banach lattices and positive operators, Grundlehren Math. Wiss., no. 215, Springer-Verlag, Berlin and New York, 1974.

12. E. Thorp and R. Whitley, The strong maximum modulus theorem for analytic functions into a Banach space, Proc. Amer. Math. Soc. 18 (1967), 640-646.

FACUlty OF TECHNICAL SCIENCES, UNIVERSity OF MARIBOR, 62000 Maribor, Smetanova 17, P. O. Box 224, YugoslaVia 